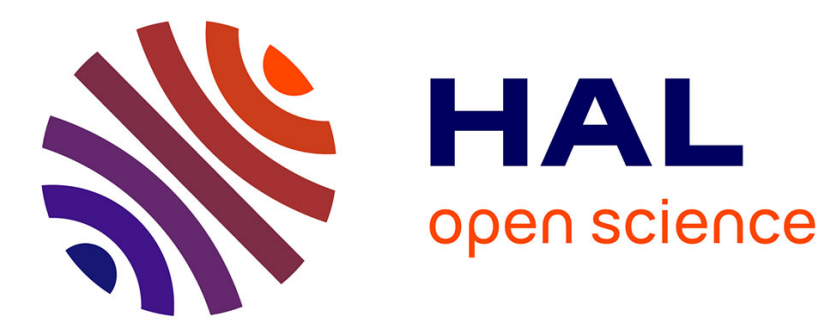

\title{
Cellules souches cancéreuses et dissémination métastatique
}

Julie Pannequin

\section{To cite this version:}

Julie Pannequin. Cellules souches cancéreuses et dissémination métastatique. Bulletin du Cancer, 2017, 104 (12), pp.1091-1093. 10.1016/j.bulcan.2017.10.014 . hal-03097174

\section{HAL Id: hal-03097174 \\ https://hal.science/hal-03097174}

Submitted on 5 Jan 2021

HAL is a multi-disciplinary open access archive for the deposit and dissemination of scientific research documents, whether they are published or not. The documents may come from teaching and research institutions in France or abroad, or from public or private research centers.
L'archive ouverte pluridisciplinaire HAL, est destinée au dépôt et à la diffusion de documents scientifiques de niveau recherche, publiés ou non, émanant des établissements d'enseignement et de recherche français ou étrangers, des laboratoires publics ou privés. 


\section{Cellules souches cancéreuses et dissémination métastatique}

\section{Cancer stem cells and metastatic dissemination}

Julie Pannequin

IGF, Univ. Montpellier, CNRS, INSERM, Montpellier, France

Annuellement dans le monde 8 millions de personnes décèdent d'un cancer et pour plus de 90\% d'entre eux ce décès serait attribué à la formation et à la progression de la maladie métastatique (1). Ce chiffre dramatique reflète notre faible connaissance des étapes clé conduisant au processus métastatique.

\section{La problématique des modèles}

La plupart des données sur le sujet ont été acquises grâce à l'utilisation de modèles murins transgéniques relativement fidèles à la pathologie humaine. Ces modèles sont bien établis, peuvent être génétiquement manipulés et sont utiles pour étudier certaines étapes de la dissémination tumorale. Cependant, même si ces données sont très importantes, les conclusions obtenues à partir de modèles murins doivent être obligatoirement validées chez l'homme et cette validation représente un véritable obstacle car les sites métastatiques sont souvent extrêmement difficiles à prélever et les rares biopsies réalisées sont généralement un mélange de métastase et de tissu environnant. Un effort considérable a été réalisé ces dernières années pour développer des méthodes et technologies afin d'isoler et de caractériser les cellules tumorales circulantes directement à partir d'échantillons sanguins de patients (2-4). Même si ces modèles sont précieux, le rendement des mises en culture demeure trop faible et le nombre de lignées disponibles trop peu important pour tirer des conclusions fiables pour le moment. 
Les premières évidences de l'existence d'un lien entre le processus métastatique et le phénotype de CSC proviennent d'observations cliniques. En effet, il a tout d'abord été montré qu'il existait une corrélation entre l'expression élevée de marqueurs de cellules souches et une récidive métastatique dans le cancer du côlon (5). Par ailleurs, les populations de cellules tumorales issues de tumeur primaire capables de générer des métastases dans des souris immunodéficientes peuvent être triées sur la base de l'expression de marqueurs de cellules souches $(6,7)$. Enfin des cellules cancéreuses avec des propriétés de cellules souches ont été caractérisées directement à partir d'échantillons sanguins de patients $(2,8)$.

Le processus métastatique, très dépendant des mécanismes de transitions entre les phénotypes épithéliaux et mésenchymateux (EMT et TEM), inclut l'invasion et l'intravasion des cellules de la tumeur primaire, la dissémination de ces cellules dans la circulation, l'extravasion dans divers organes, la survie de ces cellules à l'arrivée dans l'organe, l'entrée en phase de latence/dormance, la réactivation et la colonisation finale pour générer des métastases macroscopiques (Figure 1). L'ensemble de ces étapes est étroitement lié au phénotype de cellules souches cancéreuses et la suite de cet article sera consacrée à la description de certains de ces liens décrits dans la littérature. 


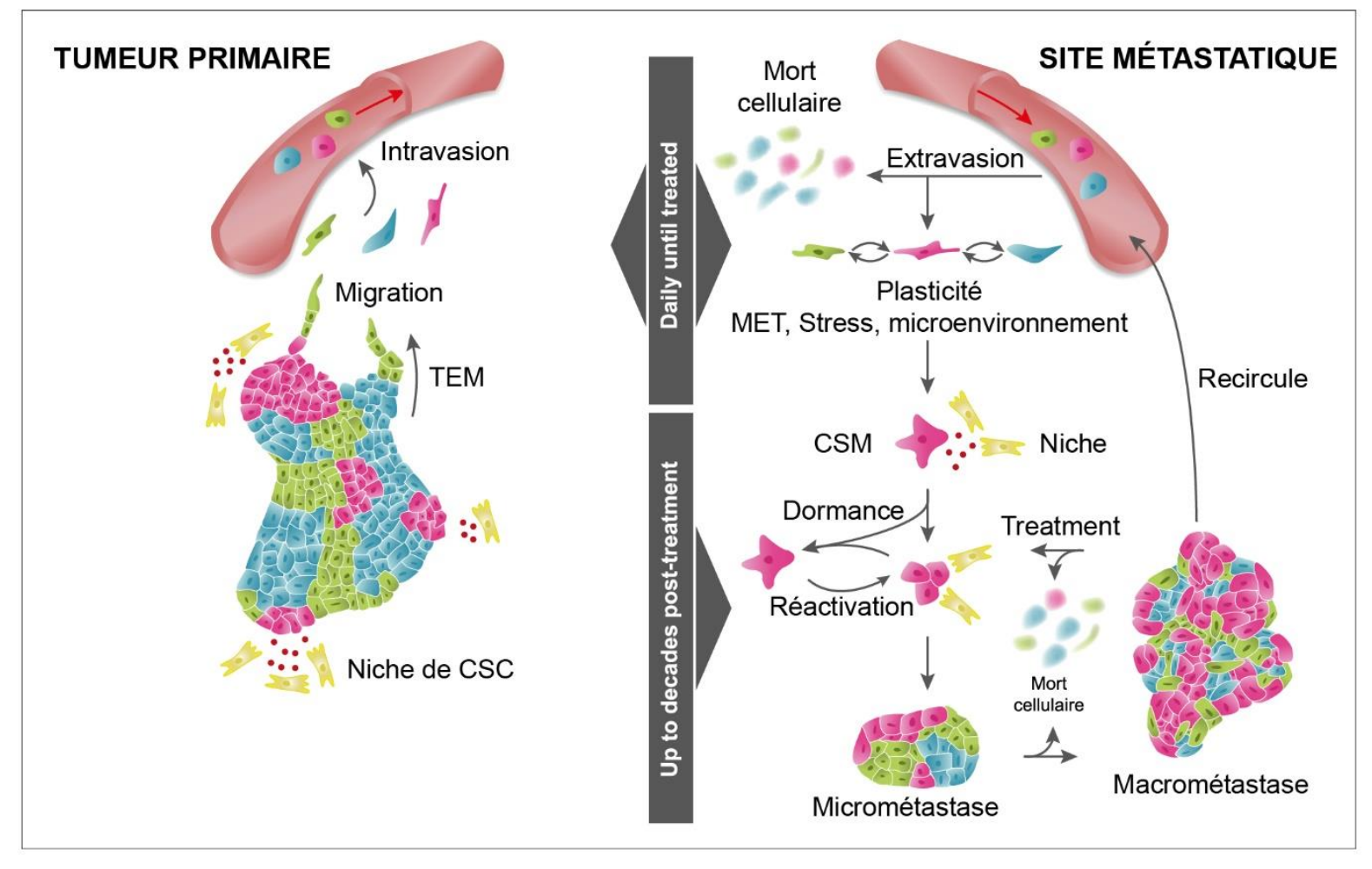

Figure 1 : Schéma du processus métastatique

\section{$\underline{E M T \text { et } C S C}$}

L’EMT (transition épithélio-mésenchymateuse) est l'ensemble des mécanismes qui permet le passage des cellules épithéliales (jointives) en cellules mésenchymateuses (migratoires). Dès les premières étapes de la dissémination tumorale, les cellules cancéreuses pourraient réactiver ce programme embryonnaire latent et c'est en 2008 pour la première fois que l'équipe de Weinberg a démontré qu'une induction forcée de l'EMT favorisait un phénotype de CSC (9). Dans ce même article, ils ont démontré que l'expression des marqueurs d'EMT était particulièrement importante dans les CSC. Quelques années plus tard, la même équipe a montré que la plasticité tumorale, permettant cette fois ci la transition entre un phénotype souche et un phénotype différencié et réciproquement, était dépendante de l'expression du facteur de transcription clé pour l'EMT : Zeb1 (10). De nombreux articles depuis ont permis de solidifier le lien entre EMT, CSC et potentiel métastatique soulignant l'intérêt de cibler 
l’EMT et/ou le phénotype souche pour prévenir de la survenue des métastases en particulier pour les patients qui présentent des stades avancés mais non métastatiques. Cependant même si cette stratégie thérapeutique est séduisante, deux obstacles persistent. Tout d'abord cibler les CSC seulement ne suffit pas puisque la plasticité tumorale permet aux non-CSC de se dédifférencier en CSC, il faut donc se débarrasser des CSC mais également des non-CSC. Le deuxième point est le fait que bloquer l'EMT favoriserait dans le même temps la MET qui représente également une étape clé dans l'implantation de la métastase dans un organe à distance.

\section{$\underline{\text { CTC et CSC }}$}

Alors que pendant des années des efforts considérables ont été réalisés afin de compter les CTC dans le sang des patients, sur la base d'expression de marqueurs spécifiques, il est maintenant crucial de les caractériser. La tâche n'est pas facile car les CTC sont extrêmement rares et hétérogènes. Si des cellules tumorales doivent posséder des propriétés de CSC ce sont certainement les cellules tumorales circulantes (CTC). En effet, ces cellules qui ont quitté la tumeur primaire doivent d'abord s'adapter à un environnement drastiquement différent et survivre en absence totale d'interaction avec la matrice, elles doivent ensuite entrer en dormance puis après une « réactivation » ces cellules doivent être capables d'initier une nouvelle tumeur dans un organe à distance possédant encore un nouveau microenvironnement. Au final il semble que moins de 0.01\% des CTC puissent accomplir le processus dans son entier et donner lieu à une métastase (11).La première publication qui a véritablement démontré que les CTC étaient douées de propriétés de CSC et fonctionnellement capables d'initier des métastases date de 2013 (8). L'an dernier, notre équipe a également décrit un phénotype souche des CTC sur des lignées établies à partir de sang de patients avec un cancer colorectal métastatique (2). Nous avons également montré que ces cellules 
étaient capables de donner des métastases dans le foie après une injection dans la rate de souris nude. Auparavant il avait été démontré des corrélations entre l'expression des marqueurs de CSC et ceux de résistance aux traitements. Par exemple, les CTC expriment des gènes codant pour des protéines de résistance aux chimiothérapies (12) et elles peuvent être dans un état de quiescence dans la circulation (13) afin de devenir dans ce cas résistantes aux traitements conventionnels ciblant les cellules prolifératives.

Dans un futur proche il va falloir s'intéresser particulièrement aux CTC avec un phénotype de CSC pour mieux comprendre ces cellules mais aussi et surtout pour les cibler car elles représenteraient les véritables «mauvaises graines » du processus métastatique.

\section{Dormance et CSC}

La notion de dormance a été suggérée dès les années 30 par le Dr Willis qui avait observé qu'une métastase pouvait émerger sur des organes à distance de nombreuses années après la résection de la tumeur primaire (13). Il a ainsi postulé que les cellules tumorales pouvaient avoir disséminé et réprimé leur prolifération pendant longtemps avant de finalement initier une métastase. La dormance nécessite donc un arrêt de la prolifération, une quiescence, et une perte du caractère tumorigène des cellules tumorales. Quelques études ont récemment démontré que le programme de dormance pour les cellules tumorales était étroitement associé au phénotype de cellules souches cancéreuses. En effet, l'expression de certains marqueurs de CSC semble être cruciale pour la programmation de l'état de dormance des cellules tumorales (14). Par ailleurs, au même titre que dans le tissu normal le caractère souche est conservé par la niche et grâce aux facteurs qui y sont secrétés. Dans ce contexte, une étude sur un modèle murin

a élégamment démontré que des cellules métastatiques de cancer de la prostate entraient en compétition avec les cellules souches hématopoiétiques pour aller se loger 
dans la moelle osseuse (15). Il y aurait ainsi des signaux de la niche métastatique qui maintiendraient les cellules dans un état souche "prolifératif » et une niche dormante qui serait essentielle pour préserver le phénotype souche quiescent des cellules en dormance. La question cruciale sur laquelle il va falloir sérieusement se pencher c'est ce qui déclenche le passage d'un état non prolifératif «dormant» à un état prolifératif «tumorigène » pour une cellule cancéreuse. Les modèles in vitro pour essayer de répondre à cette question même s'ils sont précieux ne sont pas suffisants. En effet, seules les cellules qui prolifèrent vont survivre en culture et en plus ce modèle s'affranchit complètement du microenvironnement qui semble primordial au maintien de l'un ou l'autre des phénotypes. Enfin les modèles murins ne sont pas satisfaisants. Par exemple sur les souris xénogreffées, les rares cellules qui pourraient être en dormance ne seraient pas détectées avec la sensibilité de la luminescence actuelle par exemple et le problème est assez similaire avec les modèles transgéniques développant spontanément des métastases.

En conclusion de ce chapitre, nous pouvons dire que le lien entre CSC et dissémination tumorale est maintenant bien accepté dans la littérature mais que des efforts considérables sont maintenant à fournir afin de générer de nouveaux modèles murins qui récapitulent avec le plus de fidélité les étapes clé de la pathologie humaine avec un focus particulier sur la dormance qui est un sujet d'étude trop peu étudié au jour d'aujourd'hui. Enfin l'accès à des échantillons tumoraux humains provenant de différents compartiments (sang, foie, poumon, os, moelle osseuse..) grâce aux collaborations avec les cliniciens est également crucial pour avancer dans ce domaine et pour espérer diminuer le nombre dramatique de décès causé par les métastases. 
1. Talmadge JE, Fidler IJ. AACR centennial series: the biology of cancer metastasis: historical perspective. Cancer Res. 15 juill 2010;70(14):5649-69.

2. Grillet F, Bayet E, Villeronce O, Zappia L, Lagerqvist EL, Lunke S, et al. Circulating tumour cells from patients with colorectal cancer have cancer stem cell hallmarks in ex vivo culture. Gut. oct 2017;66(10):1802-10.

3. Bobek V, Gurlich R, Eliasova P, Kolostova K. Circulating tumor cells in pancreatic cancer patients: enrichment and cultivation. World J Gastroenterol. 7 déc 2014;20(45):17163-70.

4. Yu M, Bardia A, Aceto N, Bersani F, Madden MW, Donaldson MC, et al. Cancer therapy. Ex vivo culture of circulating breast tumor cells for individualized testing of drug susceptibility. Science. 11 juill 2014;345(6193):216-20.

5. Merlos-Suárez A, Barriga FM, Jung P, Iglesias M, Céspedes MV, Rossell D, et al. The intestinal stem cell signature identifies colorectal cancer stem cells and predicts disease relapse. Cell Stem Cell. 6 mai 2011;8(5):511-24.

6. Malanchi I, Santamaria-Martínez A, Susanto E, Peng H, Lehr H-A, Delaloye J-F, et al. Interactions between cancer stem cells and their niche govern metastatic colonization. Nature. 7 déc 2011;481(7379):85-9.

7. Pang R, Law WL, Chu ACY, Poon JT, Lam CSC, Chow AKM, et al. A subpopulation of CD26+ cancer stem cells with metastatic capacity in human colorectal cancer. Cell Stem Cell. 4 juin 2010;6(6):603-15.

8. Baccelli I, Schneeweiss A, Riethdorf S, Stenzinger A, Schillert A, Vogel V, et al. Identification of a population of blood circulating tumor cells from breast cancer patients that initiates metastasis in a xenograft assay. Nat Biotechnol. juin 2013;31(6):539-44.

9. Mani SA, Guo W, Liao M-J, Eaton EN, Ayyanan A, Zhou AY, et al. The epithelialmesenchymal transition generates cells with properties of stem cells. Cell. 16 mai 2008;133(4):704-15.

10. Chaffer CL, Marjanovic ND, Lee T, Bell G, Kleer CG, Reinhardt F, et al. Poised chromatin at the ZEB1 promoter enables breast cancer cell plasticity and enhances tumorigenicity. Cell. 3 juill 2013;154(1):61-74.

11. Oskarsson T, Batlle E, Massagué J. Metastatic stem cells: sources, niches, and vital pathways. Cell Stem Cell. 6 mars 2014;14(3):306-21.

12. Gazzaniga $P$, Gradilone A, Petracca A, Nicolazzo C, Raimondi C, Iacovelli $R$, et al. Molecular markers in circulating tumour cells from metastatic colorectal cancer patients. J Cell Mol Med. août 2010;14(8):2073-7.

13. Hadfield G. The dormant cancer cell. Br Med J. 11 sept 1954;2(4888):607-10. 
14. Sosa MS. Dormancy programs as emerging antimetastasis therapeutic alternatives. Mol Cell Oncol. janv 2016;3(1):e1029062.

15. Shiozawa Y, Pedersen EA, Havens AM, Jung Y, Mishra A, Joseph J, et al. Human prostate cancer metastases target the hematopoietic stem cell niche to establish footholds in mouse bone marrow. J Clin Invest. avr 2011;121(4):1298-312. 